\title{
Time-Aware CF and Temporal Association Rule-Based Personalized Hybrid Recommender System
}

\author{
Dan Yang, University of Science and Technology LiaoNing, Anshan, China \\ Zheng Tie Nie, Northeastern University, Shenyang, China \\ Fajun Yang, Nanyang Technological University, Jurong West, Singapore
}

\begin{abstract}
Most recommender systems usually combine several recommendation methods to enhance the recommendation accuracy. Collaborative filtering $(\mathrm{CF})$ is a best-known personalized recommendation technique. While temporal association rule-based recommendation algorithm can discover users' latent interests with time-specific leveraging historical behavior data without domain knowledge. The concept-drifting and user interest-drifting are two key problems affecting the recommendation performance. Aiming at the above problems, a time-aware $\mathrm{CF}$ and temporal association rule-based personalized hybrid recommender system, TP-HR, is proposed. The proposed time-aware CF algorithm considers evolving features of users' historical feedback. And time-aware users' similar neighbors selecting measure and time-aware item rating prediction function are proposed to keep track of the dynamics of users' preferences. The proposed temporal association rule-based recommendation algorithm considers the time context of users' historical behaviors when mining effective temporal association rules. Experimental results on real datasets show the feasibility and performance improvement of the proposed hybrid recommender system compared to other baseline approaches.
\end{abstract}

\section{KEYWORDS}

CF, Hybrid Recommender System, Temporal Association Rule, Time-Aware

\section{INTRODUCTION}

Recommender systems (Adomavicius, G., \& Tuzhilin, A., 2005) have been developed and researched more than 20 years. Various recommendation methods and techniques have been proposed including demographic, content based, collaborative filtering (CF) (Breese et al., 1998), knowledge based (Wu et al., 2018), utility based, association rule based (Ghoshal, A., \& Sarkar, S., 2014; Ghoshal et al., 2015), and deep neural network based recommendation (Bai et al., 2017) recently. Currently almost all search engines and e-commerce websites have widely applied recommendation algorithms to bring better user experience and huge economic benefits. And cloud service provider needs to recommend its services to users (Qi et al., 2017). CF algorithm is a successful widely used technique 
in recommender systems which primarily works by aggregating and ranking the preferences of users with similar past behaviors. However users' preferences and interests are not static but changing over time. Traditional user-based CF algorithm does not consider the effect of temporal information of users' historical behaviors on user similarity, not consider the users' interests changing over time on item rating prediction, which seriously affect the recommendation performance. In order to solve these problems, this paper proposes a time-aware user-based CF algorithm introducing time-aware similar neighbors selecting measure to find neighborhood set which is most similar to the interests and preferences of the target user. In most cases, a single recommendation algorithm has its advantages and disadvantages in different specific application scenarios. Usually most commercial recommender systems use a combination of various recommendation algorithms to form hybrid recommender systems to improve the recommendation accuracy. $\mathrm{CF}$ algorithm has the problem of sparsity. Temporal association rule based recommendation algorithm can discover users' latent interests with time specific leveraging users' historical behavior data without domain knowledge. Therefore, this paper proposes a personalized hybrid recommender system $T P-H R$ based on time-aware CF and temporal association rule based recommendation algorithm.

The major contribution of this paper can be summarized as:

- A comparative study of various existing time-aware recommender systems and hybrid recommender systems;

- We propose the time-aware personalized hybrid recommender system $T P-H R$ by combining time-aware user-based $\mathrm{CF}$ algorithm and temporal association rule based recommendation algorithm which not only capture temporal dynamics of users' interests over time and considers users' time context to recommend;

- We propose the time-aware CF algorithm to improve the quality of CF recommendation, introducing the time decay function to the user-based CF algorithm when calculating similar users for the target user. Moreover we propose the time-aware item rating prediction function;

- Find a suitable temporal association rule based recommendation algorithm taking into account the time context of users' behaviors to improve the recommendation accuracy;

- Comparative performance analysis of proposed $T P-H R$ on real datasets with other baselines.

The remainder of the paper is structured as follows. Section II discusses related works reported in the literature. Section III presents the $T P-H R$ framework. Section IV introduces our proposed time-aware $\mathrm{CF}$ algorithm in detail. Section $\mathrm{V}$ gives recommendation algorithm based on temporal association rule in detail. Experimental results are presented in Section VI, and Section VII concludes the paper.

\section{RELATED WORK}

In this section, we first introduce related work on time-aware recommender systems, and then association rule mining and temporal association rule mining, and finally hybrid recommender systems, which related to our algorithms.

Time-aware recommender systems (Campos, P. G., Díez, F., \& Cantador, I., 2014 \& Zhong, 2015\&Qi, 2016) are becoming more and more concerned in academic and industrial recently which can be seen as special cases of context-aware recommender systems(Liu et al., 2010). The importance of context information has also been widely studied recently in the field of recommender systems. Traditional people tend to focus only on the relationships between users and items, and less on their context such as time, location, surrounding people, emotions, activity states, and network conditions. Context-aware recommender systems further improve recommendation accuracy and user satisfaction by introducing context information. Some of the existing related works take the temporal information 
into consideration in user modeling and recommendation strategies. Related work (Ding, Y., \& Li, X., 2005) uses a CF of time decay function to recommend time perception. Related work (Ding et al., 2006) proposes a new CF considering concept drift. A CF based on matrix factorization with temporal dynamics is proposed (Koren, \& Yehuda., 2009). User's bias and item's bias based on time function are used in the grading prediction step. Related work (Baltrunas, L., \& Amatriain, X., 2009) uses a repeat pattern in the user's time to divide a user's profile into a time dependent recommendation of a micro portrait at multiple intervals. Related work (Toni et al., 2010) proposes a music recommender system that considers time context. Related work (Lathia et al., 2009) proposes to automatically update the size of each user's neighbors based on K Nearest Neighbors of CF, rather than using a fixed size k. Related work (Xiang et al., 2010) proposes a session-based Temporal Graph (STG) which models users' long-term and short-term preferences over time. Related work (Wei et al., 2012) extends the neighborhood based CF algorithm to track user interests and item popularity over time by incorporating two kinds of temporal information for making timely recommendations. Related work (Liu et al., 2013) makes use of social network information and time information for context aware movie recommendation. Related work (Song et al., 2016) proposes a deep neural network model, which enhances the recommendation performance by combining long-term static preferences and short-term temporal preferences. Recently with the surge of deep learning, deep learning methods such as convolutional neural network (CNN) (Yuan, et al., 2017) and recurrent neural network (RNN) are used to solve sequential recommendation (Niu, 2017) problem. Related work (Li et al., 2017) leverages product graph embedding model to do time-aware production recommendation. Related work (Tang J.X., \& Wang K. 2018) studies personalized Top-N sequential recommendation problem using a convolutional sequence embedding recommendation model.

\subsection{Association Rule Mining and Temporal Association Rule Mining}

Association rule mining (Agrawal et al., 1993) and temporal association rule mining (Rainsford, C. P., \& Roddick, J. F., 1999; Yang, H., \& Yang, C. C., 2015; Matthews, S. G., Gongora, M. A., Hopgood, A. A., \& Ahmadi, S., 2013) have first been studied in the transactional databases and temporal databases. The goal of temporal association rule mining or sequential association rule mining (Harms, S. K., \& Deogun, J. S., 2004) is to discover meaningful sequential patterns in the occurrence of itemsets and their temporal relationships. Related work (Ozden et al., 1998) studies cyclic association rules that occur periodically over time. Related work (Chen, X. \& Petrounias, I., 1999) studies the temporal features for association rules (e.g., time intervals the rule holds). The concept of temporal support (Ale, J. M., \& Rossi G. H., 2000) is introduced and temporal information as constraints is used in the rules discovery process. Related work (Kam, P. S., \& Fu, W. C., 2000) studies several events patterns paired with temporal relations including before, after and during. Graph temporal association rules (Namaki et al, 2017) are proposed to detect regularities between events in temporal graph.

\subsection{Hybrid Recommender Systems}

This combines different recommendation algorithms and recommend according to different mixing strategies. Generally speaking, a single recommendation algorithm has its own advantages and disadvantages. So in practice a variety of recommendation algorithms are often used to recommend a hybrid strategy utilizing auxiliary information of items or users about recommendation. A hybrid web recommender system (Wanaskar et al., 2013) based on improved association rule mining algorithm recommends websites to users. In order to overcome the problems of cold start and data sparsity in CF (Qi et al.,2017), many related works introduce additional information based on CF, and propose hybrid recommendation algorithms, such as combination of content-based recommendation and CF. Related work (Jamali, M., \& Ester, M., 2010) uses social networks to improve the lookup of similar neighbors in CF. Related work (Kouki et al., 2015) proposes a generic hybrid recommender system framework HyPER. Related work (Chen et al., 2016) contains the user evaluation of the text phraselevel emotional analysis to recommend. Our proposed hybrid recommender system $T P$ - $H R$ combines 
time-aware user-based $\mathrm{CF}$ algorithm and temporal association rule mining algorithm. Our focus is on leveraging temporal information in both algorithms to capture users' dynamic preferences and make time-aware recommendation.

\section{TIME-AWARE PERSONALIZED HYBRID RECOMMENDER SYSTEM}

In this section we first give some preliminary related to personalized recommendation algorithm, then lay out framework of our proposed personalized hybrid recommender system.

\subsection{Preliminary}

User-item feedback matrix with timestamp $\boldsymbol{R}^{t}$ : With m users $U=\left\{u_{1}, \ldots, u_{m}\right\}$ and $\mathrm{n}$ items $I=\left\{i_{1}\right.$, $\left.\ldots, i_{n}\right\}$, the user behaviors (positive primary feedbacks) to item, i.e., $M \times N$ user-item matrix with timestamp $R^{t}$ is defined as follows:

$R_{k j}^{t}=\left\{\begin{array}{l}1 \text { or positive integer, if }\left(u_{k}, i_{j}\right) \text { interaction is observed } \\ 0, \text { otherwise }\end{array}\right.$

where, value 1 or positive integer in $R^{t}$ represents interactions at timestamp $t$ between users and items, e.g., a user watched a movie and rated it or user browsed a restaurant website. Similarly value 0 means a mixture of negative feedback at timestamp $t$ (the users are not interested in the items) and unobserved interactions (the users are not aware of these items for now).

Each value in the matrix $R^{t}$ can be seen as a 4-tuple of the form $\langle u, i, r, t>$, where $u$ is the user, $i$ is the item, $r$ represents user $u$ 's preference (rating) on $i$, and t represents the timestamp of the opinion. For example, $<$ user $_{1}$, item ${ }_{3}, 4,2017-1-6>$ represents that the rating rated by user ${ }_{1}$ for item ${ }_{3}$ at 2017-1-6 is 4. The recommendation task can be defined as a prediction problem which aims to infer the value of the interaction label of user-item pair $\langle u, i>$.

\subsection{TP-HR Overview}

The proposed framework of time-aware $\mathrm{CF}$ and temporal association rule based personalized hybrid recommender system $T P-H R$ is shown in Figure 1. It considers the temporal features of users' behaviors in user-based CF algorithm to improve prediction accuracy. It introduces time decay function to user-item matrix $R^{t}$ when selecting neighbors for the target user. Moreover it combines temporal association rule based recommendation to find some latent interesting items for users to enhance the recommendation accuracy.

\section{TIME-AWARE USER-BASED CF ALGORITHM}

The main idea of our time-aware user-based CF algorithm is to add some appropriate time decay function for the user feedbacks (i.e., ratings) in $R^{t}$ in order that the recently feedbacks can contribute more to the similar user calculation for the target user. In this section, we first introduce the time decay function, and then the time-aware user similarity measure. Finally, we give the time-aware item rating prediction function.

\subsection{Time Decay Function}

Traditional user-based CF algorithm ignores the temporal information of users' historical behaviors and treats all the historical data the same weight and importance. However expired data or data of a long time ago not only cannot reflect the current interests of users, also affect accuracy of user similarity 


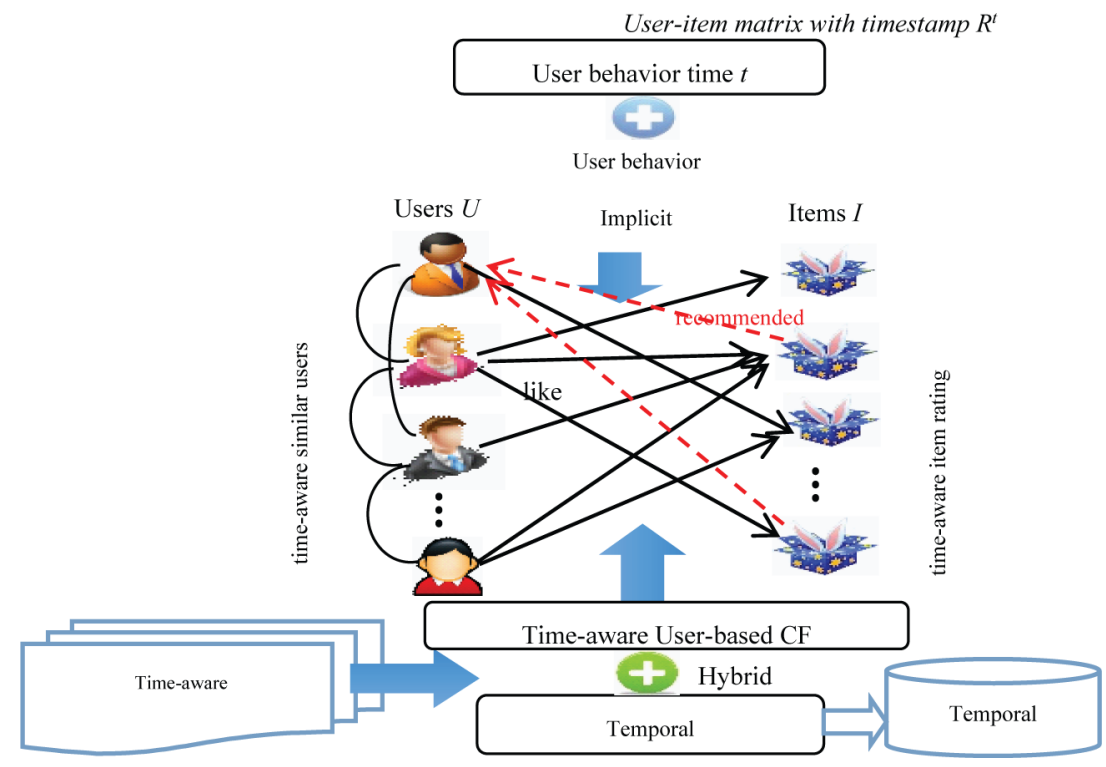

calculation and item rating prediction. The traditional user-based $\mathrm{CF}$ algorithm without time-aware feature cannot satisfy the user's dynamic demand of recommendation. So we introduce the time decay function (Ding, Y., \& Li, X., 2005) (formula 2, 3) to our proposed time-aware CF algorithm:

$f(\Delta t)=e^{-\lambda \cdot \Delta t}$

$\lambda=\frac{1}{T_{0}}$

where, $\mathrm{T}_{0}$ is the half-life parameter, and $\lambda$ is the decay rate. The value of $f(\Delta t)$ is in the range of $(0,1)$, and it reduces with time. However, there are other classic time decay functions such as logistic function.

\subsection{Time-Aware User Similarity Measure}

The main idea of user-based CF is to collect similar users' choices in the past to recommend items for the target user. That is to say similar users will have common preferences. So to choose similar users for the target user accurately is very important for the user-based CF recommendation performance. The intuition here is that the more recent data in $R^{t}$, the more it contributes to select similar users for the target user to enhance the quality of selected neighbors. In the similarity of user u and user $v, u$ 's neighborhood $N(u)$, according to the timestamp of the user behavior of user rating score, introducing the time decay function $f(\Delta t)$, by which the older data have the lower weight, the more recent data have the higher weight. Here we adopt the popular Pearson Correlation Coefficient to calculate the similarity between users by introducing time decay function. The time-aware user similarity calculation formula for the target user $u$ and other user $v$ (formula 4) is as follows: 


$$
\operatorname{sim}_{t}(u, v)=\frac{\sum_{i \in u(I) \cap v(I)}\left(r_{u, i}-\bar{r}_{u}\right)\left(r_{v, i}-\bar{r}_{v}\right) f\left(t_{u i}\right) f\left(t_{v i}\right)}{\sqrt{\sum_{i \in u(I)}\left(r_{u, i}-\bar{r}_{u}\right)^{2} f\left(t_{u i}\right)} \sqrt{\sum_{i \in v(I)}\left(r_{v, i}-\bar{r}_{v}\right)^{2} f\left(t_{v i}\right)}}
$$

where, $t_{v i}$ represents the time interval between user $v$ and item $i$ and the time interval of the current time $\left(t_{u i}\right)$. $t_{u i}$ indicates the time interval between user $u$ and the current time of item $i$. And $u(I), v(I)$ represent item sets feedbacked by user $u$, user $v$ respectively.

Select Top-K users with high time-aware similarity scores as the neighbors set $N(u)$ of the target user $u$.

\subsection{Time-Aware Item Rating Prediction}

We assume that the more recent data of $N(u)$ has greater impact on item rating prediction for the target user $u$, i.e, user preferences are time-aware. The intuition here is that the more recent data of similar users of the target user, i.e., the more recent behaviors, the more it contributes to item rating prediction. Then prediction of user $\mathrm{u}$ on unknown item $i$ can be calculated by formula 5:

$\operatorname{pre}_{t}(u, i)=\bar{r}_{u}+\frac{\sum_{v \in N(u)} \operatorname{sim}_{t}(u, v)^{*}\left(r_{v, i}-\overline{r_{v}}\right)}{\sum_{v \in N(u)} \operatorname{sim}_{t}(u, v)}$

where $\bar{r}_{u}$ is the average rating of user $u$ for all the items rated by $u, \bar{r}_{v}$ is the average rating of user $v$ for all the items rated by $v, N(u)$ is similar neighbors of user $u$, and $\operatorname{sim}_{t}(u, v)$ is the time-aware user similarity of user $u$ and $v$ according to the formula (4).

Once the predicted scores of items are obtained, K-nearest neighbors (KNN) algorithm is used to get Top-K items as the recommendation list from time-aware user-based $\mathrm{CF}$ algorithm.

\section{TEMPORL ASSOCIATION RULE BASED RECOMMENDATION AND PERSONALIZDE HYBRID RECOMMENDATION ALGORITHM}

When the data in $R^{t}$ is sparse, the accuracy of the Pearson correlation coefficient calculation is reduced. And it will affect the recommendation accuracy to a great extent. Therefore, it is necessary to discover users' potential interesting items based on temporal association rule for personalized recommendation. In this section, we first introduce association rule and temporal association rule, and then temporal association rule mining based recommendation algorithm, and finally the proposed personalized hybrid recommendation algorithm.

\subsection{Association Rule and Temporal Association Rule}

Association rule is used for discovering interesting relations between variables in large databases. Association rule mining is a general data mining method. It is intended to identify strong rules discovered in databases. Let $I=\left\{i_{1}, i_{2}, \ldots, i_{m}\right\}$ be the item set, an association rule is defined as the form:

Association Rule: $A \rightarrow B$

where $A \subset I, B \subset I, A \cap B=\phi$. For example, the rule \{onions,potatoe $\rightarrow$ \{burger $\}$ found in the sales data of a supermarket would indicate that if a customer buys onions and potatoes together, he/she is likely to also buy burger. The strength of a rule is measured by its support and confidence. 


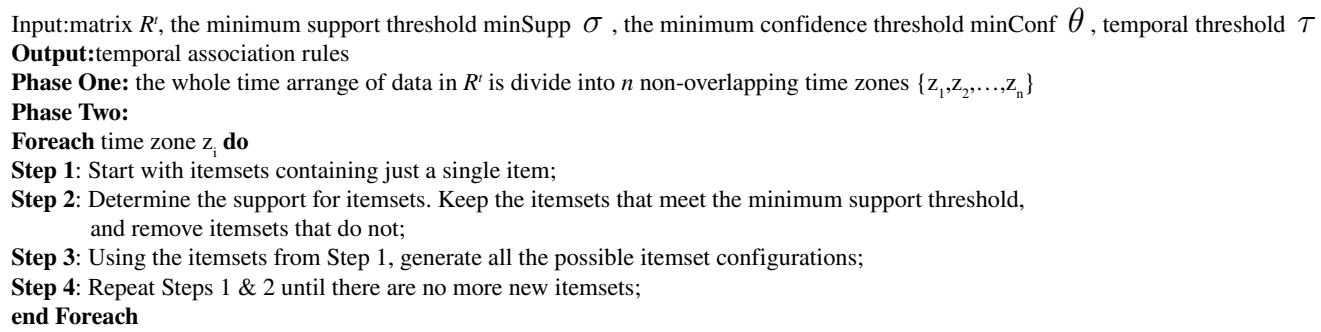

Temporal association rule states "if items $\mathrm{X}$ exist in transactions at a specified time, then items $\mathrm{Y}$ are likely to exist in the same set of transactions". To discover temporal associations within a time window between two events e 1 and $e_{2}$ rather than association rules at specific timestamps. Such rules mean "if event $\mathrm{e}_{1}$ occurs, then event $\mathrm{e}_{2}$ is likely to occur in $\Delta t$ time." Let $T_{\mathrm{I}}=\left\{t i_{1}, t i_{2}, \ldots\right\}$ be a set of time intervals, countable infinite:

Temporal association Rule: $X \rightarrow Y$ [time interval ti]

where $X \subset I, Y \subset I, X \cap Y=\phi$. Temporal association rule can indicate interesting relationships between itemsets with temporal information $t$. For example, the temporal association rule $\{$ Titanic $\} \rightarrow$ TTransformers: Age of Extinction\}[weekend] indicates if a user sees the movie Titanic also likely to see the movie transformer: age of extinction. The temporal association rule $\{$ bread $\} \rightarrow\{$ milk $\}[7: 30 \mathrm{AM}-8: 30 \mathrm{AM}]$ means that if a customer buys bread also likely to buy milk at 7AM to $8 \mathrm{AM}$.

Traditional association rule mining seldom considers the temporal dynamics or the time sequence of transactions. And association rule is mined in the whole time range of transactions. However association rule based recommendation is time-dependent. Each association rule has specified time range of itself. When considering temporal information the rules will be more meaningful and valuable to recommendation. So temporal association rule mining is used for time-aware recommendation that holds for specified time or time interval, with the additional expressive power to capture the time context of recommender system.

\subsection{Temporal Association Rule Based Recommendation Algorithm}

When association rule is used in a recommendation algorithm, in the formula (6), A represents an item that a user who has scored or has positive feedback. B represents an item that a user never gives score or feedback. The meaning of the rule is that if item A appears in the target user's historical behaviors, then item B will appear in a recommended list for the target user with some probability. Recommendation based on temporal association rule will also generate corresponding recommendation list. The recommended weight of each item in the recommended column should be measured by two values: minimum support $\min \operatorname{Supp}(A \cup B)$ and minimum confidence minConf(B|A). The proposed temporal association rule based recommendation algorithm pseudo code is shown as follows. The algorithm is based on the best known association rule mining algorithm Apriori by introducing the temporal constraints. First the whole time arrange of data in $R^{t}$ is divided into $n$ non-overlapping time zones. Then we use association rule mining algorithm in each time zones and discover the time domain of the association rules to get temporal association rules. Finally make recommendation according to the time context $t$ of user. 


\subsection{Time-Aware Personalized Hybrid Recommendation Algorithm}

The recommended item list $R_{1}$ is obtained from our time-aware $\mathrm{CF}$ algorithm. And recommended item list $R_{2}$ is obtained from our temporal association rule based recommendation algorithm. Currently we simply de-duplication the union of $R_{1}$ and $R_{2} R_{1} \cup R_{2}$ to get the final recommended item list $R L$ for the target user. In the future work we will leverage some machine learning method to learn the ranking of $R_{1} \cup R_{2}$. The pseudo code of our proposed personalized hybrid recommendation algorithm is shown as follows.

\section{EXPERIMENTS}

The experiments are conducted on a $3.5 \mathrm{GHz}$ Pentium 4 Windows machine with 8 GB memory and $500 \mathrm{~GB}$ of hard disk.

\subsection{Datasets and Evaluation Metrics}

Datasets: The MovieLens datasets include movie ratings, movie metadata (title, genre and release date) and demographic information about the users. Users' ratings range from 1 to 5 , with halfstar increments. The statistics information of the experimental datasets is shown in Table 1.

Evaluation metrics: We evaluate the $T P-H R$ with Mean Absolute Error MAE (formula 8) which is a popular metric in $\mathrm{CF}$ as follows:

$M A E=\frac{\sum_{u, i \in R}\left|P_{u, i}-R_{u, i}\right|}{|I|}$

where, $P_{u, i}$ denotes user u's rating prediction on item $i, R_{u, i}$ denotes user u's true rating for item $i$, and the $|I|$ represents the total number of the items. A smaller MAE value means a better performance of recommendation algorithm.

\section{Algorithm 2. Time-aware Personalized Hybrid Recommendation Algorithm}

Input:user-item feedback matrix with timestamp $R^{t}$, target user $u$, user $u$ 's time context $t$

Output:recommended item list for $u R L$

Step 1: Time-aware user similarity calculation for user $u$ : $\operatorname{sim}_{t}(u, v)$;

Step 2: Select Top-K most similar users as neighborhood of user $u: N(u) ; / / \mathrm{k}$ is the number of neighbors

Step 3: Time-aware rating prediction of item $i$ : $\operatorname{pre}_{t}(u, i)$;

Step 4: Generate recommendation list $R_{1}$ from time-aware CF;

Step 5: Find temporal frequent itemsets by temporal association rule mining algorithm according to $t$;

Step 6: Generate recommendation list $R_{2}$ from temporal association rule based recommendation algorithm;

Step 7: $R L \longleftarrow$ de-duplication of $R_{1} \cup R_{2}$.

Table 1. Statistics of Experimental Datasets

\begin{tabular}{|l|l|l|l|l|l|l|}
\hline \multicolumn{1}{|c|}{ Dataset } & \#Users & \#Movies & \#Ratings & \multicolumn{1}{c|}{$\begin{array}{c}\text { Avg.ratings } \\
\text { per user }\end{array}$} & $\begin{array}{c}\text { Time interval } \\
\text { (days) }\end{array}$ & $\begin{array}{c}\text { Sparsity } \\
(\%)\end{array}$ \\
\hline MovieLens-100K & 1000 & 1700 & 100,000 & 20 & 215 & 94.1 \\
\hline MovieLens-1M & 6000 & 4000 & $1,000,000$ & 20 & 4665 & 95.8 \\
\hline
\end{tabular}




\subsection{Experimental Results and Analysis}

Impact of the number of neighbors: The number of neighbors is varying from 15 to 120 and $\mathrm{T}_{0}$ value is varying from 20, 40 to 80, MAE values using different $\mathrm{T}_{0}$ on both datasets are presented in Figure 2.

From Figure 2 it is observed that MAE values are reduced with different values and the number of neighbors. With the increasing of the number of neighbors, the MAE values show a declining trend. When the number of neighbors is 75 and $T_{0}$ is 20 , the minimum MAE value is gotten. Then with the increasing of the number of neighbors MAE values present increasing trend again.

Impact of parameter $\mathbf{T}_{\mathbf{0}}$ : Table 2 shows different MAE values with different $\mathrm{T}_{0}$ values when the number of neighbors is 75 . The lower the value of $\mathrm{T}_{0}$, the larger decay rate and the lower the importance of the historical behaviors compared to more recent data. We can see from Table 2 that parameter $\mathrm{T}_{0}$ influences the recommendation performance.

\subsection{Experimental Results and Analysis}

- Performance of time-aware CF algorithm: The movies prediction scores are calculated by the proposed time-aware user-based CF algorithm. Table 3 gives example of the recommended movie list of our time-aware user-based CF algorithm.

- Comparison using different $\mathbf{C F}$ algorithms: Compare our time-aware $\mathrm{CF}$ algorithm with other two different $\mathrm{CF}$ algorithms on two datasets:

- CF: standard user-based CF algorithm;

- Time weight CF: time weight item-based CF algorithm proposed in (Ding, Y., \& Li, X., 2005).

Figure 2. MAE using Different Neighbors and $\mathrm{T}_{0}$

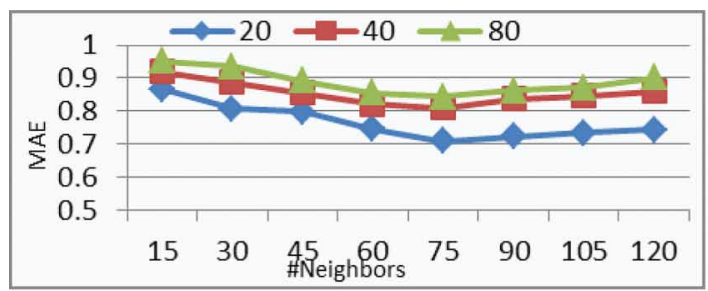

(a) MovieLens-100k

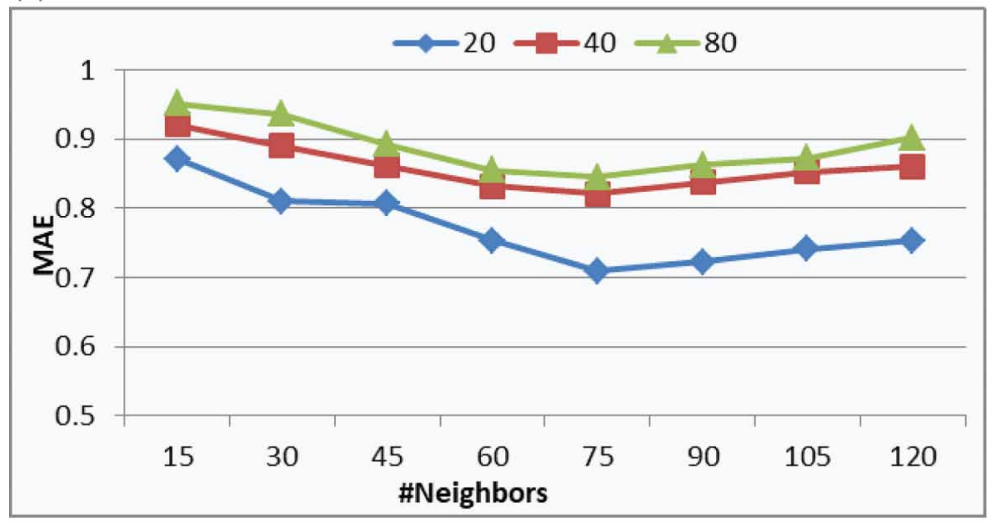

(b) MovieLens-1M 
Table 2. MAE with Different $\lambda$ and $\mathrm{T}_{0}$

\begin{tabular}{|l|l|l|}
\hline \multicolumn{1}{|c|}{ T $_{\mathbf{0}}$} & \multicolumn{1}{|c|}{$\lambda$} & \multicolumn{1}{c|}{ MAE } \\
\hline 10 & 0.10 & 0.7125 \\
\hline 20 & 0.05 & 0.7021 \\
\hline 50 & 0.02 & 0.7554 \\
\hline 100 & 0.01 & 0.7198 \\
\hline 200 & 0.005 & 0.7083 \\
\hline
\end{tabular}

Table 3. Example of Recommended Movie list of Time-aware CF

\begin{tabular}{|c|c|c|c|c|}
\hline user Id & movie list & movie Id & prediction & Avg. prediction \\
\hline \multirow{5}{*}{1} & 1 & 1500 & 4.24772577608237 & \multirow{5}{*}{3.7448} \\
\hline & 2 & 187 & 3.62048034799765 & \\
\hline & 3 & 318 & 3.61906991794361 & \\
\hline & 4 & 215 & 3.6188715768679 & \\
\hline & 5 & 192 & 3.61767254023576 & \\
\hline \multirow{5}{*}{2} & 1 & 1302 & 4.4808898148189 & \multirow{5}{*}{4.1723} \\
\hline & 2 & 1173 & 4.24313768136426 & \\
\hline & 3 & 1380 & 4.07374925130006 & \\
\hline & 4 & 1251 & 4.04386718826148 & \\
\hline & 5 & 1292 & 4.02317221746578 & \\
\hline \multirow{5}{*}{7} & 1 & 904 & 4.85345720976501 & \multirow{5}{*}{4.4664} \\
\hline & 2 & 899 & 4.84689191624404 & \\
\hline & 3 & 749 & 4.31512907667338 & \\
\hline & 4 & 306 & 4.2220141732142 & \\
\hline & 5 & 1024 & 4.09479830166807 & \\
\hline
\end{tabular}

The detailed comparison results on two datasets are shown in Figure 3. It is observed that our time-aware $\mathrm{CF}$ algorithm has smaller MAE values than the other two $\mathrm{CF}$ algorithms in the case of the number of neighbor changes. Time-aware CF considers temporal dynamics both in similar user selecting and in item rating prediction; the recommendation accuracy has been significantly improved than the other two CF algorithms. With the number of neighbors increasing, the MAE values of all the algorithms show a declining trend. When the number of neighbors is 60 , the minimum MAE value is gotten. Then MAE values increase with the increasing of the number of neighbors.

Performance of temporal association rule based recommendation algorithm: Table 4 and Table

5 give the results of Top- 5 recommended movie list generated by temporal frequent 1 -itemsets and temporal frequent 2-itemsets respectively on MovieLens-100K. 


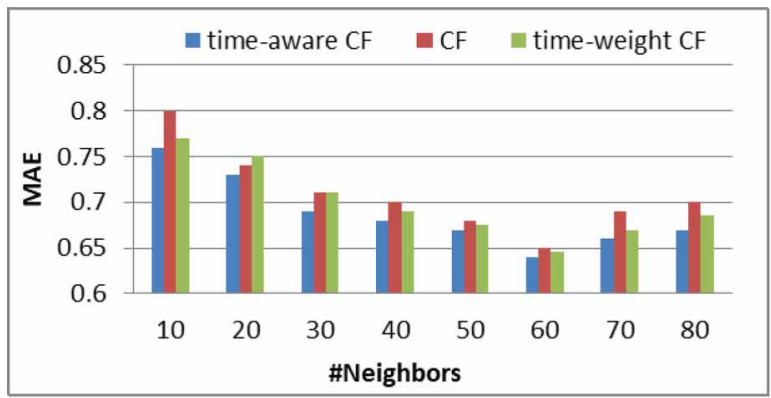

(a) MovieLens-100k

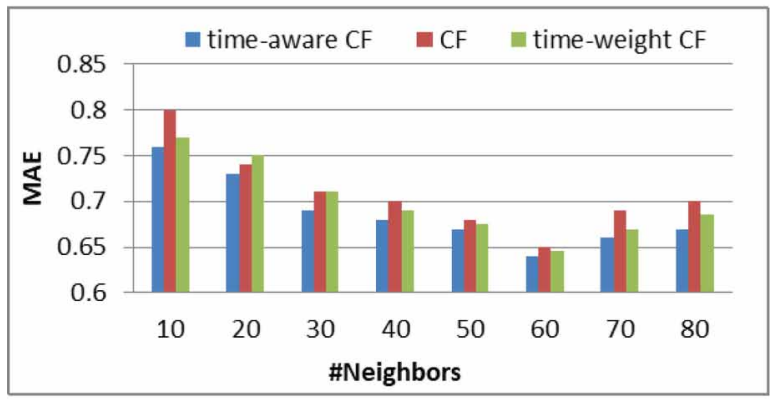

(b) MovieLens-1M

From Table 4 and Table 5, it is observed that the average confidence of temporal frequent 1 -itemsets is between 0.6553 and 0.7417 and the average confidence of temporal frequent 2-itemsets is between 0.7939 and 0.8745 . The results show that temporal association rule based recommendation can avoid the low accuracy problem of user-based CF algorithm and still recommend the higher interesting items for the target user. Moreover, the confidence of temporal frequent 2-itemsets is higher than those of temporal frequent 1-itemsets.

Performance of $\boldsymbol{T P}-\boldsymbol{H R}$ : We compare TP-HR with the following baselines on two datasets:

- Baseline1: user-based CF

- Baseline2: hybrid algorithm based on user-based CF and association rule mining

- Baseline3: hybrid algorithm based on time-aware user-based CF and association rule mining

- Baseline4: hybrid algorithm based on user-based CF and temporal association mining

The detailed experimental results are shown in Figure 4, by comparing the MAE values of the $T P-H R$ and those of baselines, it is observed that TP-HR based on time-aware user-based CF and temporal association rule studied in this paper is highly advanced and executable in recommendation accuracy than other baselines. Moreover we can find that $T P-H R$ performs best though the users' rating data is very sparse (from $94.1 \%$ to $95.8 \%$ ). The results indicate that $T P$ - $H R$ provides a good trade-off between efficiency and accuracy.

\section{CONCLUSION}

This paper presents a personalized hybrid recommender system $T P$ - $H R$ based on time-aware userbased $C F$ algorithm and temporal association rule based recommendation algorithm. $T P-H R$ captures 
Table 4. Part of Recommendation List of Temporal Frequent 1-itemsets

\begin{tabular}{|c|c|c|c|c|c|}
\hline movie Id & movie list & movie Id & Confidence & Avg. Conf & time interval \\
\hline \multirow{5}{*}{4} & 1 & 50 & 0.825 & \multirow{5}{*}{0.7417} & \multirow{5}{*}{ weekday } \\
\hline & 2 & 56 & 0.7416666666666667 & & \\
\hline & 3 & 98 & 0.708333333333333 & & \\
\hline & 4 & 79 & 0.6916666666666667 & & \\
\hline & 5 & 100 & 0.658333333333333 & & \\
\hline \multirow{5}{*}{7} & 1 & 50 & 0.718875502008032 & \multirow{5}{*}{0.6765} & \multirow{5}{*}{ weekday } \\
\hline & 2 & 100 & 0.654618473895582 & & \\
\hline & 3 & 98 & 0.57429718875502 & & \\
\hline & 4 & 56 & 0.562248995983936 & & \\
\hline & 5 & 174 & 0.558232931726908 & & \\
\hline \multirow{5}{*}{11} & 1 & 174 & 0.757763975155279 & \multirow{5}{*}{0.6553} & \multirow{5}{*}{ weekend } \\
\hline & 2 & 172 & 0.645962732919255 & & \\
\hline & 3 & 181 & 0.62111801242236 & & \\
\hline & 4 & 195 & 0.596273291925466 & & \\
\hline & 5 & 127 & 0.583850931677019 & & \\
\hline
\end{tabular}

Table 5. Part of Recommendation List of Temporal Frequent 2-itemsets

\begin{tabular}{|c|c|c|c|c|c|}
\hline movie Id & movie list & movie Id & confidence & Avg. Conf & time interval \\
\hline \multirow{5}{*}{14} & 1 & 50 & 0.935483870967742 & \multirow{5}{*}{0.8745} & \multirow{5}{*}{ weekday } \\
\hline & 2 & 174 & 0.887096774193548 & & \\
\hline & 3 & 98 & 0.854838709677419 & & \\
\hline & 4 & 172 & 0.82258064516129 & & \\
\hline & 5 & 174 & 0.806451612903226 & & \\
\hline \multirow{5}{*}{78} & 1 & 50 & 0.824324324324324 & \multirow{5}{*}{0.7939} & \multirow{5}{*}{ weekday } \\
\hline & 2 & 56 & 0.797297297297297 & & \\
\hline & 3 & 100 & 0.783783783783784 & & \\
\hline & 4 & 174 & 0.77027027027027 & & \\
\hline & 5 & 79 & 0.716216216216216 & & \\
\hline \multirow{5}{*}{$\begin{array}{l}12 \\
96\end{array}$} & 1 & 174 & 0.839285714285714 & \multirow{5}{*}{0.8080} & \multirow{5}{*}{ weekend } \\
\hline & 2 & 50 & 0.8125 & & \\
\hline & 3 & 56 & 0.794642857142857 & & \\
\hline & 4 & 98 & 0.785714285714286 & & \\
\hline & 5 & 174 & 0.723214285714286 & & \\
\hline
\end{tabular}

the temporal information of users' historical data in both algorithms and makes their combination impacts on time-aware recommendation to improve recommendation accuracy. In contrast to previous work, time decay function is introduced both in user's similar neighbors selecting and item rating 
Figure 4. Comparison with Different Recommendation Algorithms on Two Datasets w.r.t Two Sparse Settings

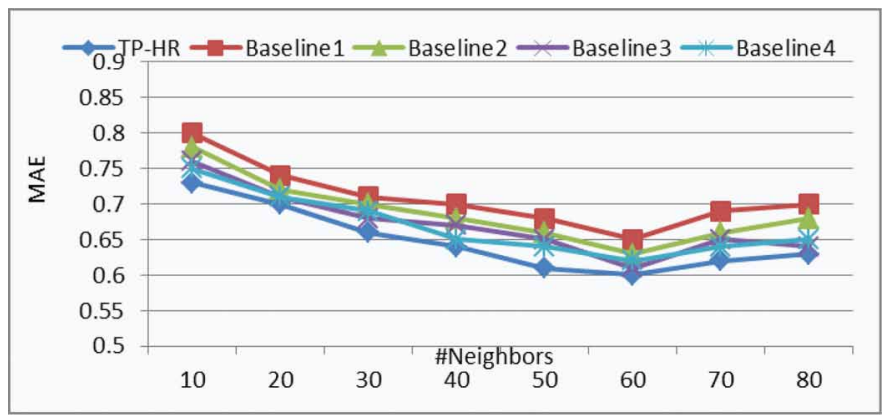

(a) MovieLens-100k

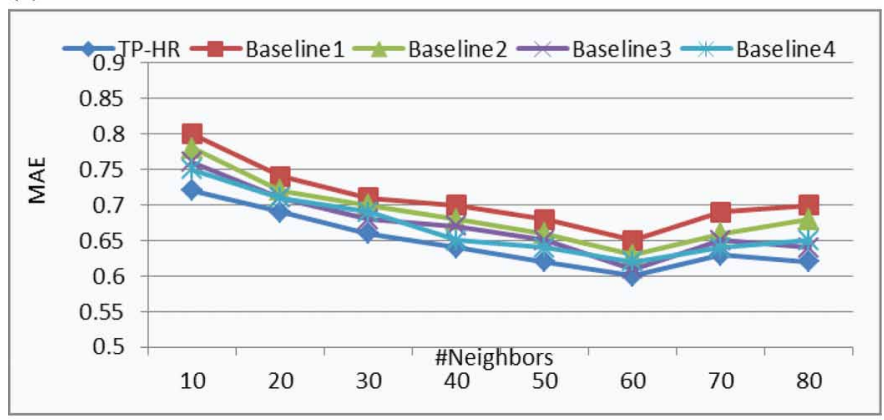

(b) MovieLens-1M

prediction for the target user. The time-aware user-based CF algorithm overcomes the limitations of native $\mathrm{CF}$ approaches and improves prediction performance. The temporal association rule based recommendation algorithm can find latent new interesting items for users with specified temporal information. The experimental results on real datasets show that TP-HR leads to more accurate recommendation and provides a good trade-off between recommendation efficiency and accuracy.

As for the future work, we consider some machine learning methods to re-rank recommendation results of the hybrid recommender system to obtain the final recommendation list. Also we continue to explore more advanced time-aware user similarity measure to find similar neighbors for the target user. Meanwhile we will explore the temporal diversity of the time-aware hybrid recommender system.

\section{ACKNOWLEDGMENT}

This research was supported by the National Natural Science Foundation of China (Grant NO. 61672142); the Natural Science Foundation of Liaoning province, China (Grant NO. 20170540471). 


\section{REFERENCES}

Adomavicius, G., \& Tuzhilin, A. (2005). Toward the next generation of recommender systems: a survey of the state-of-the-art and possible extensions. IEEE Transactions on Knowledge and Data Engineering, 17(6), 734-749. doi:10.1109/TKDE.2005.99

Agrawal, R., Imieliński, T., \& Swami, A. (1993). Mining association rules between sets of items in large databases. In ACM SIGMOD International Conference on Management of Data (Vol. 22, pp.207-216). ACM. doi:10.1145/170036.170072

Ale, J. M., \& Rossi, G. H. (2000). An approach to discovering temporal association rules. In ACM Symposium on Applied Computing (pp. 294-300). DBLP. doi:10.1145/335603.335770

Bai, T., Wen, J. R., Zhang, J., \& Zhao, W. X. (2017). A Neural Collaborative Filtering Model with Interactionbased Neighborhood. ACM. doi:10.1145/3132847.3133083

Baltrunas, L., \& Amatriain, X. (2009). Towards time-dependant recommendation based on implicit feedback. Workshop on Context-Aware Recommender Systems.

Campos, P. G., Díez, F., \& Cantado, I. (2014). Time-aware recommender systems: A comprehensive survey and analysis of existing evaluation protocols. User Modeling and User-Adapted Interaction, 24(1-2), 67-119. doi:10.1007/s11257-012-9136-x

Carsâ, Ă. Z., Breese, J. S., Heckerman, D., \& Kadie, C. (1998). Empirical analysis of predictive algorithms for collaborative filtering. In Fourteenth Conference on Uncertainty in Artificial Intelligence (Vol. 7, pp. 43-52). Morgan Kaufmann Publishers Inc.

Cebrián, T., Villegas, P., \& Amatriain, X. (2010). Music recommendations with temporal context awareness. In ACM Conference on Recommender Systems (pp. 349-352). ACM. doi:10.1145/1864708.1864786

Chen, X., \& Petrounias, I. (1999). Mining temporal features in association rules. Academic Press.

Chen, X., Qin, Z., Zhang, Y., \& Xu, T. (2016). Learning to Rank Features for Recommendation over Multiple Categories. In International ACM SIGIR Conference on Research \& Development in Information Retrieval (pp.305-314). ACM. doi:10.1145/2911451.2911549

Ding, Y., \& Li, X. (2005). Time weight collaborative filtering. Academic Press.

Ding, Y., Li, X., \& Orlowska, M. E. (2006). Recency-based collaborative filtering. In Australasian Database Conference (Vol. 49, pp.99-107). Australian Computer Society, Inc.

Ghoshal, A., Menon, S., \& Sarkar, S. (2015). Recommendations using information from multiple association rules: A probabilistic approach. Information Systems Research, 26(3). doi:10.1287/isre.2015.0583

Ghoshal, A., \& Sarkar, S. (2014). Association rules for recommendations with multiple items. INFORMS Journal on Computing, 26(3), 433-448. doi:10.1287/ijoc.2013.0575

Harms, S. K., \& Deogun, J. S. (2004). Sequential association rule mining with time lags. Journal of Intelligent Information Systems, 22(1), 7-22. doi:10.1023/A:1025824629047

Jamali, M., \& Ester, M. (2010). A matrix factorization technique with trust propagation for recommendation in social networks. In ACM Conference on Recommender Systems (Vol. 45, pp.135-142). ACM. doi:10.1145/1864708.1864736

Kam, P. S., \& Fu, W. C. (2000). Discovering Temporal Patterns for Interval-based Events. Data Warehousing and Knowledge Discovery. Springer Berlin Heidelberg. doi:10.1007/3-540-44466-1_32

Koren \& Yehuda. (2009). Collaborative filtering with temporal dynamics. ACM.

Kouki, P., Fakhraei, S., Foulds, J., Eirinaki, M., \& Getoor, L. (2015). HyPER: A Flexible and Extensible Probabilistic Framework for Hybrid Recommender Systems. In ACM Conference on Recommender Systems (pp.99-106). ACM. doi:10.1145/2792838.2800175

Lathia, N., Hailes, S., \& Capra, L. (2009). Temporal collaborative filtering with adaptive neighbourhoods. In International ACM SIGIR Conference on Research and Development in Information Retrieval (pp.796-797). ACM. 
Li, Y., Chen, W., \& Yan, H. (2017). Learning Graph-based Embedding For Time-Aware Product Recommendation. ACM. doi:10.1145/3132847.3133060

Liu, N. N., Cao, B., Zhao, M., \& Yang, Q. (2010). Adapting neighborhood and matrix factorization models for context aware recommendation. ACM. doi:10.1145/1869652.1869653

Liu, N. N., He, L., \& Zhao, M. (2013). Social temporal collaborative ranking for context aware movie recommendation. ACM Transactions on Intelligent Systems and Technology, 4(1), 1-26. doi:10.1145/2414425.2414440

Matthews, S. G., Gongora, M. A., Hopgood, A. A., \& Ahmadi, S. (2013). Web usage mining with evolutionary extraction of temporal fuzzy association rules. Knowledge-Based Systems, 54(C), 66-72. doi:10.1016/j.knosys.2013.09.003

Namaki, M. H., Wu, Y., Song, Q., Lin, P., \& Ge, T. (2017). Discovering Graph Temporal Association Rules. In ACM on Conference on Information and Knowledge Management (pp.1697-1706). ACM. doi:10.1145/3132847.3133014

Niu, S., \& Zhang, R. (2017). Collaborative Sequence Prediction for Sequential Recommender. ACM. doi: $10.1145 / 3132847.3133079$

Ozden, B., Ramaswamy, S., \& Silberschatz, A. (1998). Cyclic association rules. In International Conference on Data Engineering, 1998. Proceedings (pp.412-421). IEEE. doi:10.1109/ICDE.1998.655804

Qi, L., Xu, X., Dou, W., Yu, J., Zhou, Z., \& Zhang, X. (2016, December 14). Time-aware ioe service recommendation on sparse data. Mobile Information Systems, 2016, 2016. doi:10.1155/2016/4397061

Qi, L., Zhang, X., Dou, W., \& Ni, Q. (2017). A distributed locality-sensitive hashing-based approach for cloud service recommendation from multi-source data. IEEE Journal on Selected Areas in Communications. doi:10.1109/JSAC.2017.2760458

Qi, L., Zhou, Z., Yu, J., \& Liu, Q. (2017). Data-sparsity tolerant web service recommendation approach based on improved collaborative filtering. IEICE Transactions on Information and Systems, 100(9), 2092-2099. doi:10.1587/transinf.2016EDP7490

Rainsford, C. P., \& Roddick, J. F. (1999). Adding Temporal Semantics to Association Rules. In European Conference on Principles of Data Mining and Knowledge Discovery (Vol. 1704, pp. 504-509). Springer-Verlag. doi:10.1007/978-3-540-48247-5_65

Song, Y., Elkahky, A. M., \& He, X. (2016). Multi-Rate Deep Learning for Temporal Recommendation. In International ACM SIGIR Conference on Research and Development in Information Retrieval (pp.909-912). ACM.

Tang, J. X., \& Wang, K. (2018). Personalized Top-N Sequential Recommendation via Convolutional Sequence Embedding. WSDM, 2018, 565-573. doi:10.1145/3159652.3159656

Wanaskar, U. H., Vij, S. R., \& Mukhopadhyay, D. (2013). A hybrid web recommendation system based on the improved association rule mining algorithm. Journal of Software Engineering \& Applications, 06(8), 32-36. doi:10.4236/jsea.2013.68049

Wei, S., Ye, N., \& Zhang, Q. (2012). Time-Aware Collaborative Filtering for Recommender Systems. In Chinese Conference on Pattern Recognition (Vol. 321, pp.663-670). Springer.

Wu, C., Evgeniya, Z., Chen, Y., \& Li, F. (2018). Time Optimization of Multiple Knowledge Transfers in the Big Data Environment. Computers Materials \& Continua, 54(3), 269-285.

Xiang, L., Yuan, Q., Zhao, S., Chen, L., Zhang, X., \& Yang, Q. (2010). Temporal recommendation on graphs via long- and short-term preference fusion. ACM SIGKDD Conference on Knowledge Discovery and Data Mining, 723-732. doi:10.1145/1835804.1835896

Yang, H., \& Yang, C. C. (2015). Using health-consumer-contributed data to detect adverse drug reactions by association mining with temporal analysis. ACM Transactions on Intelligent Systems \& Technology, 6(4). doi:10.1145/2700482

Yuan, C., Li, X., Wu, Q. M. J., Li, J., \& Sun, X. (2017). Fingerprint liveness detection from different fingerprint materials using convolutional neural network and principal component analysis. Computers Materials \& Continua, 53(4), 357-371.

Zhong, Y., Fan, Y., Huang, K., Tan, W., \& Zhang, J. (2015). Time-aware service recommendation for mashup creation. IEEE Transactions on Services Computing, 8(3), 356-368. doi:10.1109/TSC.2014.2381496 
Dan Yang is currently an Associated Professor in the School of Software, University of Science and Technology LiaoNing, China. Yang obtained her M.S. and Ph.D. degrees in Computer Software and Theory from Northeastern University from China. Her research interests include data integration, entity recommendation and semantic entity search. Dr. Yang was a visiting scholar in New Jersey Institute of Technology, Newark, NJ, USA from June 2015 to May 2016 supported by Chinese Scholarship Council of the Ministry of Education. Yang is a member of the CCF (China Computer Federation).

Tiezheng Nie is currently Associate Professor in the School of Computer Science and Engineering, Northeastern University, China. Nie obtained his M.S. and Ph.D. degrees in Computer Software and Theory from Northeastern University from China. His research interests include data integration and data quality. Dr. Nie was a visiting scholar in The Pennsylvania State University, USA from Sep. 2014 to Aug. 2015 supported by Chinese Scholarship Council of the Ministry of Education. Nie is a member of the CCF (China Computer Federation). He has published more than 20 Research papers in International/National Journals and conferences.

Fajun Yang received the Ph. D. degree in Mechanical Engineering from Guangdong University of Technology, China, in 2016. From 2015-2016, he was a Visiting Student with New Jersey Institute of Technology, Newark, NJ, USA. He is currently a Research Fellow with Nanyang Technological University, Singapore. He has 20+ journal and conference papers (majority in IEEE Transactions). His interests are Petri nets, production planning, discrete event systems, scheduling and control, evolutionary algorithms, and optimization. 